\title{
Further data on a weighted average model for judgment in a lifted weight task'
}

\author{
NORMAN H. ANDERSON, ${ }^{2}$ UNIVERSITY OF CALIFORNIA, San Diego \\ ANN JACOBSON, UNIVERSITY OF CALIFORNIA, LOS Angeles
}

\begin{abstract}
Ss lifted three weights in sequence with instructions to judge the average heaviness of the weights. In agreement with previous work, a recency effect was obtained, with the later weights having more influence on the judgment. In contrast to previous work, a discrepancy was found from predictions based on the hypothesis that the judgment was an average of the felt heavinesses of the weights. The discrepancy had the appearance of a response end-effect, and was eliminable by a scaling procedure based on functional measurement.
\end{abstract}

In the present experimental task, $S$ lifts several weights one after the other, and then judges their average heaviness. The theoretical hypothesis is that the judgment is an average of the felt heavinesses of the weights. For a given sequence of weights, this may be written as

$$
\mathrm{J}=\Sigma \mathrm{W}_{\mathrm{k}} \mathrm{S}_{\mathrm{k}}
$$

where $\mathbf{J}$ is the judgment for the sequence, $s_{k}$ is the felt heaviness, and $\mathrm{wk}_{\mathrm{k}}$ the relative influence of the $\mathrm{k}$-th stimulus weight in the sequence. If the $w_{k}$ sum to unity, then Equation 1 is a weighted average model with mathematical weights, $w_{k}$. Since $w_{k}$ measures the influence of the stimulus in the $k$-th serial position, the $w_{k}$ constitute a serial position curve which it is desired to estimate. The interpretation of the serial curve depends, in part, on the adequacy of Equation 1. Accordingly, a test of the averaging model is also desired.

Both purposes may be accomplished using the techniques of previous work (Anderson, 1964a, b). A numerical response is employed, and the stimulus sequences are constructed from a factorial design. In this, the factors are the serial positions, and the levels of a given factor are the stimuli presented at the given serial position.

In the present experiment, each sequence had three weights so there were three serial positions. For a given S, there were just two weights, High and Low, and these same two weights were used at each serial position. There are thus eight possible sequences of weights, and these form a $2^{3}$ design. If Equation 1 is correct, then the statistical interactions of this design are zero. Also, the difference between the two marginal means for the $\mathrm{k}$-th serial position is $\mathrm{w}_{\mathrm{k}}$ (sHigh - SLow). This quantity thus measures the total effect of the given serial position.

If there are no adaptation effects, (sHigh-sLow) will be constant across serial position. In that case, the observed difference between marginal means at Serial Position $\mathrm{k}$ is proportional to $w_{k}$. These observed differences then measure the relative influence of each serial position in the overall judgment.

A previous experiment (Anderson, 1967) with sequences of six weights gave preliminary support to this subjective averaging model. The present work was designed to extend the previous results by using several weight ranges, though with only three weights in each sequence.

\footnotetext{
METHOD

Design

In Experiment 1, there were five experimental conditions which differed in the range of weights that were used. Each range consisted of a pair of weights, denoted by $\mathrm{H}$ and $\mathrm{L}$. In the five respective conditions, the values of $\mathrm{H}$ and $\mathrm{L}$ in grams were $100 \&$ $90,110 \& 80,120 \& 70,130 \& 60$, and $140 \& 50$.

Within each condition, the $\mathrm{H}$ and $\mathrm{L}$ weights were used to produce all eight sequences of three weights. In formal terms, these eight sequences constitute a $2^{3}$ design in which the factors are the three serial positions in the sequence, and $H$ and $L$ are the two levels of each factor.
}

Two sequences of anchor stimuli were also used. In each condition, the high anchor was $10 \mathrm{~g}$ heavier than $\mathrm{H}$, and the low anchor was $10 \mathrm{~g}$ lighter than $\mathrm{L}$. Each anchor sequence consisted of three successive presentations of one of the anchor stimuli.

The basic stimulus group for each condition consisted of the eight regular sequences and the two anchor sequences. This basic group was presented three times to each $S$ following the practice period.

\section{Apparatus}

$S$ sat in front of a vertical black panel, $4 \mathrm{ft}$ square, that stood on a table. Each weight was lifted by pulling a handle a short distance downwards to a prescribed mark. The handle attached to a string that led over pulleys to the rear of the panel and terminated with a small at tached magnet.

The weights were similar cans filled with shot and clay. A small magnet was mounted on each weight and its placement produced a distinct click that was S's signal to pull. The weights were placed by $\mathrm{E}$ who was concealed behind the panel during the course of the experiment.

\section{Procedure}

After being shown the operation of the apparatus, $S$ was told to judge "the average heaviness of the group taking all three weights together." The numbers 1 and 20 were to be used for the lightest and heaviest groups, and 10 for the group of average heaviness. Ss were told to try to use all the numbers between 1 and 20 , and to spread their responses over the whole scale.

There were 20 practice sets. The first four consisted of two presentations of each anchor sequence, $S$ being told that these represented the lightest and heaviest sets of weights. The next six sets included both high and low anchor weights to get $S$ used to judging heterogeneous sets. Finally, a practice presentation of the basic stimulus group of 10 sets was given.

The experiment proper consisted of three presentations of the basic stimulus group of 10 sets. Four colored decks of $103 \times 5$ cards were used, one for each presentation, including practice. The 10 sequences were listed on the cards of each deck, and each deck was shuffled separately for each $S$ to randomize stimulus order. Short rests were given between successive presentations.

The timing varied somewhat from $\mathrm{S}$ to $\mathrm{S}$, but each trial required approximately $20 \mathrm{sec}$ with an approximate range of 17 to $22 \mathrm{sec}$. The time between the placement of the first weight and the release of the third weight was about $10 \mathrm{sec} ; 2 \mathrm{sec}$ later $\mathrm{E}$ told $\mathrm{S}$ to respond, and recorded S's response on the $3 \times 5$ card.

\section{Experiment 2}

In Experiment 1, an unexpected peak occurred at the second position of the serial curves for the three smallest weight ranges. To test whether this effect was real, two conditions were replicated, the $110 \& 80 \mathrm{~g}$, and the $120 \& 70 \mathrm{~g}$ weight ranges. A different $E$ ran the Ss, but an effort was made to keep running conditions constant. Design and procedure were the same, but there were differences of 1 or $2 \mathrm{sec}$ in trial timing.

\section{Subjects}

Ss were 84 females, volunteers who were fulfilling a course requirement in introductory psychology. Within each experiment, $12 \mathrm{Ss}$ were randomly assigned to each weight range.

\section{RESULTS}

The main results are in Table 1 in which the mean entries have been reduced to a per set basis. Two aspects of these data are of 
Table 1

Algebraic Values of Serial Position Main Effects, and Interactions of Three Weights Presented Serally in $2^{3}$ Design

\begin{tabular}{lrrrrrrrr} 
Serial & \multicolumn{8}{c}{ High and Low stimulus weights } \\
\cline { 2 - 9 } position & $140-50$ & $130-60$ & $120-70$ & $120-70^{2}$ & $110-80$ & $110-80^{\circ}$ & $100-90$ & Mean \\
\hline R(HHH) & 18.61 & 18.78 & 18.30 & 17.30 & 16.97 & 15.36 & 14.67 & 17.14 \\
First & 4.81 & 4.16 & 3.92 & 3.58 & 3.55 & 2.50 & 2.32 & 3.55 \\
Second & 5.57 & 5.10 & 4.56 & 3.51 & 4.44 & 2.11 & 2.82 & 4.02 \\
Third & 6.07 & 6.06 & 4.32 & 4.01 & 4.34 & 2.33 & 2.49 & 4.23 \\
F X S & .12 & .09 & -.25 & .56 & -.12 & -.03 & -.56 & -.03 \\
F X T & -.04 & .19 & .26 & -.14 & -.16 & .06 & -.44 & -.04 \\
S X T & .19 & .22 & .21 & .40 & .20 & .56 & -.14 & .23 \\
F X S X T & -1.14 & -.35 & -.29 & -.75 & -.52 & -.36 &. .12 & -.50 \\
\hline
\end{tabular}

aData from Experiment 2.

$b_{M e a n}$ response to $\mathrm{HHH}$ sequence.

interest: the serial position curves, and the interactions.

The serial position curves are given by the three lines labelled First, Second, and Third of Table 1. Each entry is a difference score that measures the effect of the weight at that serial position. For Condition $140-50$, for instance, the mean response is 4.81 higher when the first weight is $140 \mathrm{~g}$ than when it is $50 \mathrm{~g}$. The differential effect of these same two weights increases to 5.57 at the middle position, and to 6.07 at the last serial position.

This rise in the serial position curve for Condition 140-50 is a recency effect. Inspection of the other conditions in Table 1 shows that the predominant trend is toward recency, in agreement with previous work with this same task. The recency is prominent at the larger weight ranges, which are comparable to the range used in the previous experiment. At the smaller weight ranges, the data are somewhat irregular, though the observed effect of the weight pair is less in first than in third position in all but one case. The peak observed at the second position for the smaller weight ranges of Experiment 1 may be sampling error since it did not reappear in Experiment 2. At the lower weight ranges, it should be realized, less recency can occur since the High and Low weights are closer together.

The lower four lines of Table 1 give the interactions, and these are of interest since they test the averaging model. Because a design in the $2^{n}$ series was used, the interactions may be expressed algebraically as specific comparisons. The model implies that the true interaction scores are zero. If this is so, the observed interaction should not differ from zero except for chance variability.

However, it is clear that the three-way interaction in the last line of the table is consistently negative across the different groups. An overall analysis showed that this was significant, $\mathrm{F}=11.84, \mathrm{df}=1 / 77$. In addition, the interaction of the Second and Third serial positions was positive in all but one group, though it fell short of significance in the overall analysis.

The locus of the interaction can be seen in the left half of Fig. 1 which shows the overall response means for the eight sequences of the $2^{3}$ design. The two plots in this panel differ depending on whether the first weight of the sequence was $H$ or $L$. The pair of lines in each separate plot are nonparallel, and this nonparallelism represents an interaction of Second and Third serial positions at each level of the First position. However, since this nonparallelism is in opposite directions in the two plots, the effect actually appears as the First by Second by Third interaction in the overall analysis. The overall Second by Third interaction represents the extent to which the nonparallelism in the two plots does not cancel.

The shape of this interaction suggests as one possibility that the judgments may be too low for Sequence $\mathrm{HHH}$ and too high for Sequence LLL. Such an effect could reflect a scale of measurement that is not adequate theoretically. Psychological distance might be compressed near the ends of the judgment scale, giving rise to apparent floor and ceiling effects. Or, more generally, the scale endpoints may introduce a bias in the judgments.
It is possible, therefore, that the interaction results from shortcomings in the scale of measurement rather than from basic defects in the model. If this is true, a simple solution is available.

This general problem has been discussed in another connection (Anderson, 1962a), and the measurement-theoretical argument given there applies here without change. It may be assumed that the correct scale of measurement, if one exists, is a strictly monotone transformation of the actual scale. From this it follows, if the model is basically correct, that some monotone transformation of the observed judgments will satisfy the test of goodness of fit.

Accordingly, the data were rescaled by the following function: $\mathrm{X}^{\prime}=\mathrm{X}+\mathrm{A}(\mathrm{X}-\mathrm{b})^{3}$

where $X$ and $X^{\prime}$ are original and rescaled judgments. If $b$ is taken interior to the scale, the effect of the rescaling is to stretch the scale increasingly near its endpoints.

The values of $X$ were the sums of the three judgments of each set since these sums had been used in the previous statistical tests. The grand mean was 34.26 , and this was substituted for $b$ in Equation 2 since all conditions were about equal in mean response. Various values of the stretch coefficient, $A$, were then tested in overall analyses of variance. Simple trial and error quickly showed that the interactions disappeared for values of $A$ near .0005 , and this value was accordingly chosen. A further, linear transformation was then applied for purposes noted below.

The end result of this rescaling is shown in the right-hand panel of Fig. 1. Of the two plots, one is essentially parallel, and the other one has a slight nonparallelism. As far as these data go, therefore, the rescaling has accomplished its purpose.

To assess the rescaling procedure, a more detailed comparison between the original and the rescaled data is necessary. To facilitate such a comparison, it is desirable to equate the two sets

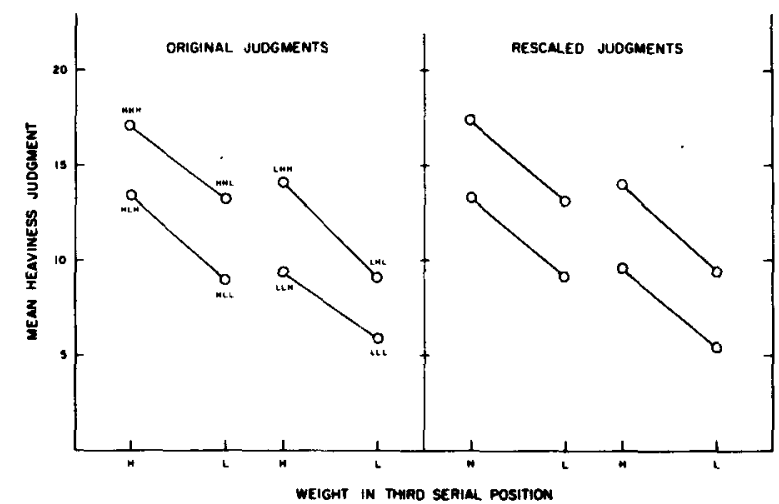

Fig. 1. Overall mean responses of original and rescaled judgments. Each pair of lines represents a two-way design based on the Second and Third serial positions of the sequence of weights. 
Table 2

Mean Squares from Analysis of Variance of Original and

Rescaled Judgments, Experiments 1 and 2 Combined

\begin{tabular}{|c|c|c|c|c|c|}
\hline \multirow[b]{2}{*}{ Source } & \multirow[b]{2}{*}{ df } & \multicolumn{2}{|c|}{ Original judgments } & \multicolumn{2}{|c|}{ Rescaled judgments } \\
\hline & & Systematic & Error ${ }^{a}$ & Systematic & Error $^{a}$ \\
\hline Weight range & 6 & 18.09 & 16.67 & 20.63 & 16.81 \\
\hline$W \times F$ & 6 & 18.92 & 2.70 & 28.73 & 3.17 \\
\hline $\mathbf{W} \times S$ & 6 & 37.72 & 3.40 & 49.34 & 3.94 \\
\hline $\mathrm{W} \times \mathrm{T}$ & 6 & 53.99 & 5.05 & 68.53 & 5.76 \\
\hline$W \times F \times S$ & 6 & 1.06 & 2.56 & 1.86 & 2.80 \\
\hline $\mathbf{W} \times \mathbf{F} \times \mathbf{T}$ & 6 & 1.35 & 2.88 & 1.40 & 3.04 \\
\hline $\mathbf{W} \times \mathbf{S} \times \mathbf{T}$ & 6 & 2.85 & 3.24 & 2.80 & 3.36 \\
\hline $\mathbf{W} \times \mathbf{F} \times \mathbf{S} \times \mathbf{T}$ & 6 & 2.77 & 3.66 & 2.07 & 3.65 \\
\hline First & 1 & 2111.80 & 2.70 & 2200.38 & 3.17 \\
\hline Second & 1 & 2706.43 & 3.40 & 2837.77 & 3.94 \\
\hline Third & 1 & 3005.93 & 5.05 & 3126.33 & 5.76 \\
\hline$F \times S$ & 1 & 0.13 & 2.56 & 0.86 & 2.80 \\
\hline $\mathbf{F} \times \mathbf{T}$ & 1 & 0.29 & 2.88 & 1.01 & 3.04 \\
\hline $\mathbf{S} \times \mathbf{T}$ & 1 & 9.05 & 3.24 & 4.43 & 3.36 \\
\hline$F \times S \times T$ & 1 & 43.34 & $3.66^{\circ}$ & 1.68 & 3.65 \\
\hline
\end{tabular}

all error terms have $77 d f$.

of data analyses in some respect since the range of the rescaled data is somewhat larger than the range of the original data. There are various criteria that may be employed, but it was decided here to equalize on error mean square for the three-way interaction since this was the critical term in the test of fit. Accordingly, the rescaled judgments were subjected to a linear transformation that equated the two given error terms, kept the grand means equal, and reduced the data to a per set basis. This linear transformation has no essential effect; in particular, all F-ratios are invariant under a linear transformation.

The two overall analyses of variance are summarized in Table 2. The critical interactions are in the last four lines of the table. The most important term is the mean square for the three-way interaction and this has been markedly reduced by the scaling procedure, from 43.34 to 1.68 . The $\mathrm{S}$ by $\mathrm{T}$ interaction is about halved and, though the other two interactions increase somewhat, none approaches its error term in size. Since these four interactions are critical for the model, it is important to note that their error terms show only small increases under the rescaling. Such increases are, of course, consistent with the increases in the main effects.

The effects of weight range and its interactions also deserve some comment. The main effect of range is not significant, reflecting the near equality of overall mean response across conditions. In this experimental design, the effects of weight range actually appear as interactions of weight range with each serial position. These are quite large and only mean that an $\mathrm{H}-\mathrm{L}$ weight pair has more effect when the weight difference is greater. Finally, the higher order interactions involving weight range remain small and not much changed by the scaling procedure. This is of interest since it indicates that the same scale transformation handles all the weight range conditions.

One final point of comparison between the original and rescaled judgments is of interest. From Fig. 1, it can be seen that the rescaling does not change the response means very much. However, these changes are accomplished by differential stretching towards the scale endpoints. The range of the original judgments was from 1 to 20; the corresponding range of the rescaled judgments was from -1.89 to 21.21 , about $20 \%$ greater.

The transformation of Equation 2 was chosen arbitrarily for convenience. A more systematic approach would use a series expansion (Anderson, 1962a), and transformations obtained in these ways will be useful in practice. However, a theoretical basis for the transformation is desirable since its final justification lies in the theory of the behavior, not in any immediate numerical success in eliminating a discrepancy.
Some related approaches are those of Bradley, Katti, and Coons (1962), Snell (1964), and Kruskal (1965). Bradley, et al, direct their efforts toward finding a scale that maximizes discriminability among a set of objects. Snell is primarily concerned to find a scale that yields normal distributions and homogeneous variances. Although both approaches have considerable interest, they have different purposes than the present model and are not directly relevant here.

However, Kruskal's (1965) article is directly relevant. It provides a method for determining a best monotone transformation for fitting a linear model subject to certain constraints, such as the constraint of zero interaction. Kruskal's basis of calculation is somewhat different, but in practice the numerical difference would probably be small. Accordingly, Kruskal's procedure may prove very useful.

\section{DISCUSSION}

Perhaps the major usefulness of the foregoing presentation is to illustrate the present measurement procedure which may, for convenience, be termed functional measurement. The logic of the procedure is straightforward, and is based on "using the postulated behavior laws to induce a scaling on the dependent variable" (Anderson, 1962a). If the model is basically correct, then it may be used as a frame on which to rescale the actual measurements. Failure to find a suitable transformation argues against the model, and success argues for it. Use of factorial designs, together with a numerical response, then provides the practical means for implementing the procedure.

On this basis, of course, the present data and analyses give some provisional support to the averaging model. Figure 1 may suggest that finding a suitable transformation was too easy, but this supports more than infirms the model. And that the same transformation applied to all the weight range conditions suggests that the rescaling may have the desired invariance over stimulus conditions.

However, there are two grounds for concern about the model, the first based on comparison with related work. The model has been reasonably successful with integration of semantic stimuli (Anderson, 1962b, 1968), and rescaling has not been needed there. However, the few studies that have used serial presentation of simple perceptual stimuli show a mixed picture. The previous experiment on weight averaging (Anderson, 1967) showed no sign of the apparent end-effect found here, possibly because of procedural differences, or because of the use of six weights rather than three. Recently completed work on length averaging has found some discrepancies with two or three lengths though not with six. On the other hand, discrepancies from the model were obtained when Ss judged the average loudness of four bursts of noise (Parducci, Thaler, \& Anderson, 1968), and the interactions had a different shape than Fig. 1. For the loudness data, the rescaling procedure had limited success, though the use of only six response categories may have given too coarse a scale.

A more general reason for concern over the model is that contrast effects (e. g., Helson, 1964) may change the value of a given weight from one sequence to another. This possibility must be kept in mind, but it may not be too serious. If context effects exist, it is not unreasonable to suppose that they are linear functions of the context stimuli (e. g., Anderson, 1966, 1967). Linear context effects would not produce statistical interactions, though they would, of course, alter the interpretation of the serial curve. Moreover, the contrast claimed for lifted weights may be largely, though not completely, non-perceptual (e. g., Anderson \& Lampel, 1965; Campbell, Lewis, \& Hunt, 1958; Parducci, 1965; Stevens, 1958) and to that extent not relevant here.

Although this discussion leaves the status of the model unsettled, it does emphasize the basic nature of the present scaling procedure. The procedure necessarily depends, of course, on the concrete experimental details since these determine the actual measurements. Simultaneously, however, it rests on and is part of the theory of the behavior. Here, for example, it would be incorrect to eliminate the discrepancies by rescaling if these arise 
from context effects. This decision rests on the substantive theory. The validation of the measurement scale depends on the validity of the theory, and conversely. This view is in the same general spirit as that of Burke (1963).

In certain respects, the present scaling procedure is similar to that employed in multidimensional scaling by Shepard (1962) and Kruskal (1964). There, as here, an arbitrary monotone transformation is applied in an attempt to force the data to meet some criterion. Multidimensional scaling, like factor analysis, employs a criterion of minimum dimensionality. In contrast, the present procedure uses a theoretical behavior law as its criterion or frame for scaling. This orientation, which is basic to the procedure, can be criticized on the ground that the resultant scales are theoryrelative. On the other hand, the procedure has the considerable advantage of being functional in the development of the theory. Moreover, it yields scales not only of the dependent, response variable, but of the independent, stimulus variables as well (Anderson, 1962a, b).

\section{REFERENCES}

ANDERSON, N. H. On the quantification of Miller's conflict theory. Psychol. Rev., 1962a, 69, 400-414.

ANDERSON, N. H. Application of an additive model to impression formation. Science, 1962b, 138, 817-818.

ANDERSON, N. H. Note on weighted sum and linear operator models. Psychon. Sci, 1964a, 1, 189-190.

ANDERSON, N. H. Test of a model for number-averaging behavior. Psychon. Sci, 1964b, 1, 191-192.

ANDERSON, N. H. Component ratings in impression formation. Psychon. Sci., 1966, 6, 279-280.

ANDERSON, N. H. Application of a weighted average model to a psychophysical averaging task. Psychon. Sci., 1967, 8, 227-228.

ANDERSON, N. H. A simple model for information integration. In R. P. Abelson, E. Aronson, W. J. McGuire, T. M. Newcomb, M. J. Rosenberg, and P. H. Tannenbaum (Eds.), Theories of cognitive consistency: $A$ sourcebook. Chicago: Rand McNally, 1968,
ANDERSON, N. H., \& LAMPEL, A. K. Effect of c itext on ratings of personality traits. Psychon. Sci, 1965, 3, 433-434.

BRADLEY, R. A., KATTI, S. K., \& COONS, I, J. Optimal scaling for ordered categories. Psychometrika, 1962, 27, 355-374.

BURKE, C. J. Measurement scales and statistical models. In M. H. Marx (Ed.), Theories in contemporary psychologv. New York: Macmillan, 1963. Pp. 147-159.

CAMPBELL, D. T., LEWIS, N. A., \& HUNT, W. A. Context effects with judgmental language that is absolute, extensive, and extra-experimentally anchored. J. exp. Psychol., 1958, 55, 220-228.

HELSON, H. Adaptation-level theory. New York: Harper \& Row, 1964.

KRUSKAL, J. B. Nonmetric multidimensional scaling: a numerical method. Psychometrika, 1964, 29, 115-129.

KRUSKAL, J. B. Analysis of factorial experiments by estimating monotone transformations of the data. J. Roy. statist. Soc., (B), 1965, 27, 251-263.

PARDUCCI, A. Category judgment: A range-frequency model. Psychol Rev., $1965,72,407-418$.

PARDUCCI, A., THALER, H., \& ANDERSON, N. H. Stimulus averaging and the context for judgment. Percept. \& Psychophys, 1968, 3, 145-150.

SHEPARD, R. N. The analysis of proximities: multidimensional scaling with an unknown distance function. Psychometrika, 1962, 27, 219-246.

SNELL, E. J. A scaling procedure for ordered categorical data. Biometrics, 1964, 20, 592-607.

STEVENS, S. S. Adaptation-level vs. the relativity of judgment. Amer. $J$. Psychol, 1958, 71, 633-646.

\section{NOTES}

1. This work was supported by National Science Foundation grants GB-3913 and GB-6666. We wish to thank Allen Parducci for the use of the weight lifting apparatus, and Anita Lampel for running Experiment 2.

2. Address, Department of Psychology, University of California, San Diego, La Jolla, Calif. 92037.

(Accepted for publication April 16, 1968.) 